\title{
An approximation for Normal Vectors of Deformable Models
}

\author{
Wu, Shin - Ting ${ }^{1}$ and Vanio Fragoso de Melo ${ }^{1,2}$ \\ ${ }^{1}$ Department of Industrial Automation and Computer Engineering (DCA) \\ School of Electrical and Computer Engineering (FEEC) \\ State University of Campinas (Unicamp) \\ P.O.Box 6101, 13083-970 - Campinas, SP, Brazil \\ \{ting,vanio\}@dca.fee.unicamp.br \\ ${ }^{2}$ Department of Mathematics and Statistics (DME) - CCT/UFCG \\ Center of Science and Technology (CCT) \\ Federal University of Campina Grande (UFCG) \\ P.O.Box 10044, 58109-970 - Campina Grande, PB, Brazil.
}

\begin{abstract}
A physically-based deformable model proposed by Terzopoulous et al. is governed by the Lagrange's form, that establishes the relation between the dynamics of deformable models under the influence of applied forces. The net instantaneous potential energy of deformation is derived on the basis of the geometric properties, namely the first and second fundamental forms. For simplicity, the normal vector at each sample point is approximated by the second derivative. In this paper we present another approximation for the normal vector which offers better visual simulation. Some comparisons are given.
\end{abstract}

\section{Introduction}

We may distinguish two types of movement for an object. The first type involves changes in position of points such that all metric properties, e.g. length, angle, area and volume of the object are preserved. The second type involves changes in the relative position of at least a pair of points which may lead to local deformation of shape. We say that an object is rigid, if it only suffers the first type of movement; otherwise, we say that it is deformable.

Terzopoulos et al. [12] proposed a deformable surface model which relates the Lagrangian motion equation with the metric and curvature tensors of the deforming surface. The metric tensors measure the deforming surface stretches and/or shears and the curvature tensors that give us the amount that a surface bends or twists while it is deforming. From this way, the physical quantities are "geometrized". Hence, in comparison with the existing approaches for cloth objects $[1,6,13,14]$, we believe that it is a potential candidate for a model which is more intuitive to use and has realistic deformation results.

Horta and $\mathrm{Wu}$ [8] perceived, however, that it is difficult to obtain with the mentioned model a deforming behavior for a surface which has a resistance against bending. Ramos et al. [11] thought that the problem derives from the lack in the control of the relationship between the metric and curvature tensors while the surface is deforming. The metric and curvature tensors must satisfy certain compatibility differential equations known as Gauss and Mainardi-Codazzi equations of surface theory [3, 9]. Nevertheless, from their exhaustive experiments, they con- cluded that, even when the compatibility equations are satisfied, unrealistic deforming behaviors concerning with bending may be yielded.

Attracted by the potentiality of the model in providing a more intuitive interface to the users, we decided to investigate more carefully the approximation of the components of the metric tensor and the components of the curvature tensors presented by Terzopoulos et al. in [12]. These components require approximations to the normal vector, the first and the second derivatives. For reducing the partial differential equation of motion into a system of linear equations, the normal vectors are replaced by the second derivatives and finite differences are applied for the derivatives. From our analysis, the direction of the normal vector, that is of fundamental importance in modeling the bending behavior, and the direction of second derivatives are seldom coincident. This motivated us to present a more precise, but still linearizable, formula for the normal vectors.

The paper is organized as follows. In section 2 some basic concepts of Differential Geometry are presented. To be self-contained, the model proposed by Terzopoulos et al. is summarized in section 3 and we show some visual effects of the deformation of surfaces that have big resistance to bending - the motivation of this work. A new approximation for the normal vector is given in section 4 . For comparison purpose, some new simulation results are presented in section 5. Finally, in section 6, some concluding remarks are drawn. 


\section{Concepts}

To be self-contained some fundamental concepts necessary for understanding this paper are given $[3,9]$.

A surface $S$ may be specified in terms of parametric equations of the form $x_{i}=x_{i}\left(a_{1}, a_{2}\right)(i=1,2,3)$, where $x_{i}$ are rectangular Cartesian coordinates of a point $P$ on the surface $S$ and $a_{j}(j=1,2)$ are parameters. We assume that the functions $x_{i}\left(a_{1}, a_{2}\right)$ are single-valued and continuous and also require that they possess as many derivatives as may be required. The position vector $\overrightarrow{\mathbf{r}}$, with rectangular Cartesian components $x_{i}$, at $P$ may be expressed as a function of the parameters $a_{i}$, where these parameters are usually called curvilinear coordinates on the surface $S$.

Let $\overrightarrow{\mathbf{r}}: \Omega \rightarrow R^{3}$ be a regular surface $S$ given by $\overrightarrow{\mathbf{r}}\left(a_{1}, a_{2}\right)=\left(x\left(a_{1}, a_{2}\right), y\left(a_{1}, a_{2}\right), z\left(a_{1}, a_{2}\right)\right), a_{1}, a_{2} \in \Omega$. As we have

$$
d \overrightarrow{\mathbf{r}}=\frac{\partial \overrightarrow{\mathbf{r}}}{\partial a_{1}} d a_{1}+\frac{\partial \overrightarrow{\mathbf{r}}}{\partial a_{2}} d a_{2},
$$

the squared length of an arc in curvilinear coordinates can be expressed by

$$
d l^{2}=d \overrightarrow{\mathbf{r}} \cdot d \overrightarrow{\mathbf{r}}=\sum_{i=1}^{2} \sum_{j=1}^{2} G_{i j} d a_{i} d a_{j},
$$

where

$$
G_{i j}(\overrightarrow{\mathbf{r}}(\overrightarrow{\mathbf{a}}))=\frac{\partial \overrightarrow{\mathbf{r}}}{\partial a_{i}} \cdot \frac{\partial \overrightarrow{\mathbf{r}}}{\partial a_{j}} .
$$

Eq.(2) is known as first fundamental form or metric tensor and the elements $G_{i j}$ are called metric coefficients. Since the inner product is symmetric, one may conclude that $G_{12}=G_{21}$.

The curvature at a given point in any curve measures the amount of variation of the tangent's direction at that point from the tangents of its neighborhood. The normal curvature of a point $P$ that lies on a curve $C$ of a surface $S$ is given by

$$
k_{n}=k \cos \theta,
$$

where $k$ is the curvature of $C$ in $P$ and $\theta$ is the angle between the vector normal to $C$ and the vector normal to $S$, in $P$.

It can be shown that

$$
k_{n}=\sum_{i=1}^{2} \sum_{j=1}^{2} B_{i j} d a_{i} d a_{j},
$$

where

$$
B_{i j}(\overrightarrow{\mathbf{r}}(\overrightarrow{\mathbf{a}}))=\overrightarrow{\mathbf{n}} \cdot \frac{\partial^{2} \overrightarrow{\mathbf{r}}}{\partial a_{i} \cdot \partial a_{j}}
$$

with $\overrightarrow{\mathbf{n}}=\left(\frac{\partial \overrightarrow{\mathbf{r}}}{\partial a_{1}} \times \frac{\partial \overrightarrow{\mathbf{r}}}{\partial a_{2}}\right) /\left\|\frac{\partial \overrightarrow{\mathbf{r}}}{\partial a_{1}} \cdot \frac{\partial \overrightarrow{\mathbf{r}}}{\partial a_{2}}\right\|$.
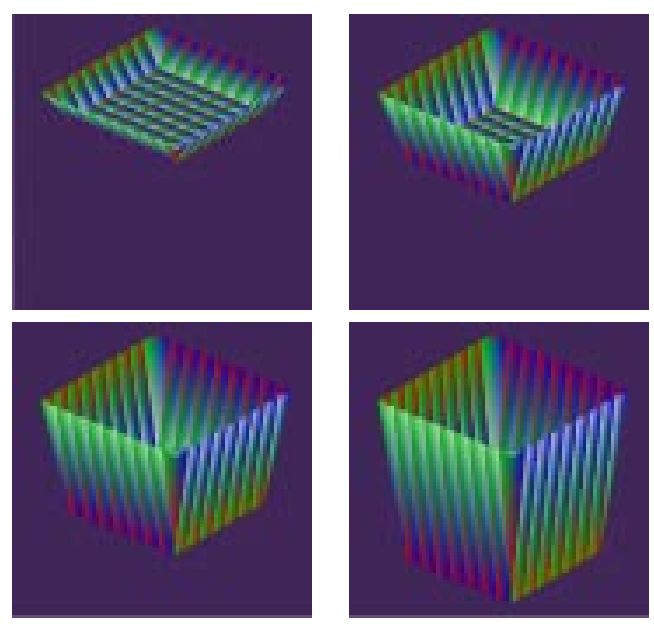

Figure 1: No resistance to metric variation and to curving.

Eq. (5) is called second fundamental form or curvature tensor and the elements $B_{i j}$ are called curvature coefficients. Since $\partial^{2}(\overrightarrow{\mathbf{r}}) / \partial a_{1} . \partial a_{2}=\partial^{2}(\overrightarrow{\mathbf{r}}) / \partial a_{2} . \partial a_{1}$, it follows that $B_{12}=B_{21}$. The plane that passes through $P \in S$ and coincides with $\frac{\partial \overrightarrow{\mathbf{r}}}{\partial a_{1}}$ and $\frac{\partial \overrightarrow{\mathbf{r}}}{\partial a_{2}}$ is called tangent plane to $S$ at $P$ and will be denoted by $T_{P}(S)$.

Roughly speaking, the coefficients $G_{i j}$ of the first fundamental form are related to the measures of an arc length, angles, and areas on a surface (metric tensor), whereas the second fundamental form $B_{i j}$ describes how the curves on a surface bend and/or twist (curvature tensor). The metric and the curvature tensors are not independent: they must satisfy certain compatibility differential equations known as Gauss and Mainardi-Codazzi equations of surface theory.

Since $\partial(\overrightarrow{\mathbf{n}}) / \partial a_{1}$ and $\partial(\overrightarrow{\mathbf{n}}) / \partial a_{2}$ at $P$ belong to $T_{P}(S)$, we may also write

$$
\frac{\partial \overrightarrow{\mathbf{n}}}{\partial a_{i}}=d_{1 i} \frac{\partial \overrightarrow{\mathbf{r}}}{\partial a_{1}}+d_{2 i} \frac{\partial \overrightarrow{\mathbf{r}}}{\partial a_{2}}, i=1,2,
$$

where

$$
\begin{aligned}
d_{i i} & =\frac{B_{12} G_{12}-B_{i i} G_{k k}}{G_{11} G_{22}-\left(G_{12}\right)^{2}}, i=1, k=2 \text { or } i=2, k=1 \\
d_{i j} & =\frac{B_{j j} G_{12}-B_{12} G_{j j}}{G_{11} G_{22}-\left(G_{12}\right)^{2}}, i \neq j
\end{aligned}
$$

$d_{i j}$ are known as Weingarten coefficients.

If $S$ is orientable, it is possible to assign to each point a natural basis given by the vectors $\partial(\overrightarrow{\mathbf{r}}) / \partial a_{1}$ and $\partial(\overrightarrow{\mathbf{r}}) / \partial a_{2}$ and $\overrightarrow{\mathbf{n}}$. By expressing the derivatives of $\partial(\overrightarrow{\mathbf{r}}) / \partial a_{1}$ and $\partial(\overrightarrow{\mathbf{r}}) / \partial a_{2}$ in the basis $\left\{\partial(\overrightarrow{\mathbf{r}}) / \partial a_{1}, \partial(\overrightarrow{\mathbf{r}}) / \partial a_{2}, \overrightarrow{\mathbf{n}}\right\}$, we obtain the formulae of Gauss

$$
\frac{\partial^{2} \overrightarrow{\mathbf{r}}}{\partial a_{i} . \partial a_{j}}=\sum_{k=1}^{2} \Gamma_{i j}^{k} \frac{\partial \overrightarrow{\mathbf{r}}}{\partial a_{k}}+B_{i j} \vec{n}, i, j=1,2,
$$



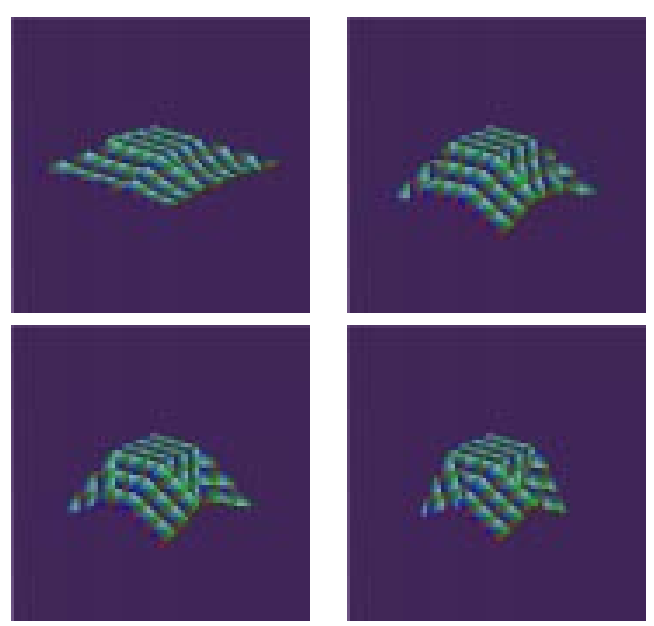

Figure 2: Resistance to metric variation and no resistance to curving.

where $\Gamma_{i j}^{k}$ are the Christoffel symbols of the surface $\overrightarrow{\mathbf{r}}\left(a_{1}, a_{2}\right)$. Since $\partial^{2} \overrightarrow{\mathbf{r}} / \partial a_{1} . \partial a_{2}=\partial^{2} \overrightarrow{\mathbf{r}} / \partial a_{2} . \partial a_{1}$, one can conclude that $\Gamma_{12}^{1}=\Gamma_{21}^{1}$ and $\Gamma_{12}^{2}=\Gamma_{21}^{2}$.

The Christoffel symbols may be derived, in their turn, with use of the second derivatives and the reciprocal vectors $\overrightarrow{\mathbf{r}}^{k}$

$$
\Gamma_{i j}^{k}=\frac{\partial^{2} \overrightarrow{\mathbf{r}}}{\partial a_{i} \partial a_{j}} \cdot \overrightarrow{\mathbf{r}}^{k}
$$

A vector $\overrightarrow{\mathbf{r}}^{i}$ is called reciprocal vector of $\frac{\partial \overrightarrow{\mathbf{r}}}{\partial a_{j}}$, if it satisfies

$$
\overrightarrow{\mathbf{r}}^{i} \cdot \frac{\partial \overrightarrow{\mathbf{r}}}{\partial a_{j}}=\delta_{j}^{i}
$$

where $\delta_{j}^{i}$ is the Kronecker delta. Using the reciprocal base system, we can write the differential of $\overrightarrow{\mathbf{r}}$, in the form of $d \overrightarrow{\mathbf{r}}=\sum_{i=1}^{2} \overrightarrow{\mathbf{r}}^{i} d x_{i}$, where $d x_{i}$ are the appropriate components of $d \overrightarrow{\mathbf{r}}$, and define the components of the reciprocal metric tensor or conjugate metric tensor

$$
G^{i j}=\overrightarrow{\mathbf{r}}^{i} \cdot \overrightarrow{\mathbf{r}}^{j},
$$

computed in terms of the metric tensors $G_{i j}$ by

$$
G^{11}=\frac{G_{22}}{\mathbf{G}}, G^{12}=G^{21}=-\frac{G_{12}}{\mathbf{G}}, G^{22}=\frac{G_{11}}{\mathbf{G}}
$$

with $\mathbf{G}=G_{11} G_{22}-G_{12} G_{21}$. It follows that the reciprocal vectors can be computed by the expression

$$
\overrightarrow{\mathbf{r}}^{i}=\sum_{j=1}^{2} G^{i j} \frac{\partial \overrightarrow{\mathbf{r}}}{\partial a_{j}}
$$

Replacing $\overrightarrow{\mathbf{r}}^{k}$ in Eq.(10) by Eq.(14), we get the following expression for the Christoffel symbols in terms of the
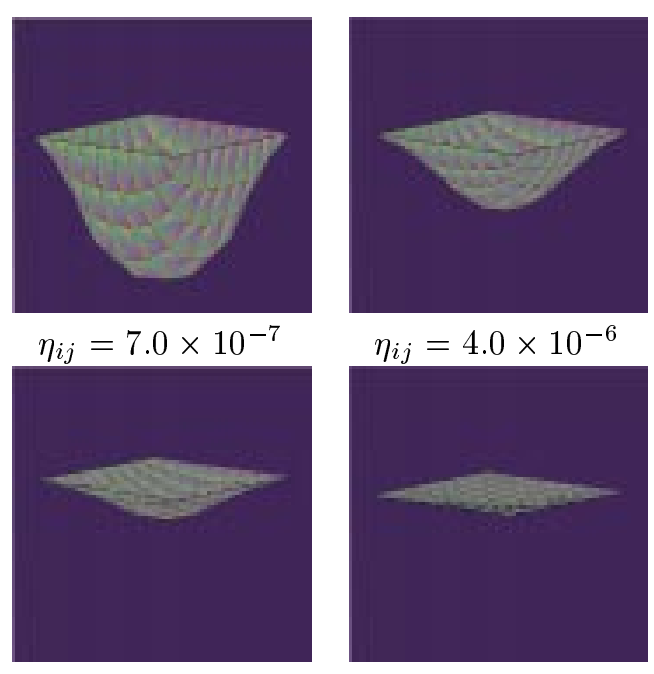

$\eta_{i j}=3.0 \times 10^{-5}$

$\eta_{i j}=1.0 \times 10^{-2}$

Figure 3: No resistance to curving and resistance to metric variation.

reciprocal metric tensors and the first and second derivatives

$$
\Gamma_{i j}^{k}=\sum_{l=1}^{2} G^{k l}\left(\frac{\partial^{2} \overrightarrow{\mathbf{r}}}{\partial a_{i} \partial a_{j}} \cdot \frac{\partial \overrightarrow{\mathbf{r}}}{\partial a_{l}}\right) .
$$

\section{A Deformable Surface Model}

The dynamics of the physically based model proposed by Terzopoulos et al. are ruled by the equation of motion in its Lagrangian formulation:

$$
\mu \frac{\partial^{2} \vec{r}}{\partial t^{2}}+\gamma \frac{\partial \vec{r}}{\partial t}+\frac{\delta \epsilon(\vec{r})}{\delta \vec{r}}=\vec{f}(\vec{r}, t) .
$$

In Eq.(16) $\mu$ is the mass density and $\gamma$ is the dumping constant at a point $\vec{r}$. The vector $\vec{f}$ denotes the total contribution of external forces at $\vec{r}$ in an instant $t$. The term corresponding to the internal energies accumulated due to elastical deformation $\varepsilon(\vec{r})$ is estimated from the following empirical consideration [12]:

$$
\epsilon(\overrightarrow{\mathbf{r}})=\int_{\Omega}\left\|\mathbf{G}-\mathbf{G}^{0}\right\|_{\alpha}^{2}+\left\|\mathbf{B}-\mathbf{B}^{0}\right\|_{\beta}^{2} d a_{1} d a_{2},
$$

which takes, for the metric and curvature tensors, a weighted norm of the difference between the deformed state and rest state values. That measure can reasonably estimate the elastical energy of a surface, attaching the amount of energy to the variations in the surface's geometric shape. In other words, that norm is a measure of the energy needed to displace the surface's points, defined over a region $\Omega$, from their rest state.

By applying the weighted norms of Eq.(17), the following simplified deformation energy is obtained: 

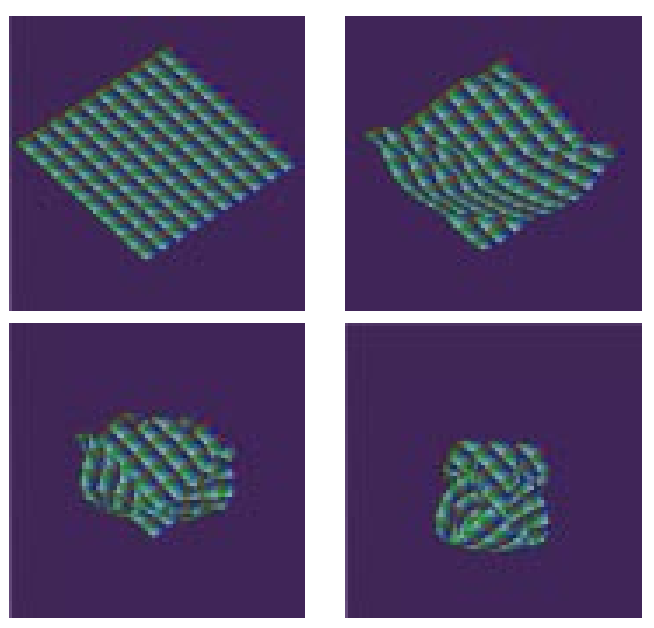

Figure 4: Resistance to curving and to metric variation.

$$
\epsilon(\overrightarrow{\mathbf{r}})=\int_{\Omega} \sum_{i, j}\left(\eta_{i j}\left(G_{i j}-G_{i j}^{0}\right)^{2}+\xi_{i j}\left(B_{i j}-B_{i j}^{0}\right)^{2}\right) d a_{1} d a_{2}
$$

The weights $\eta_{i j}$ and $\xi_{i j}$ are denominated elasticity parameters.

From Eq.(17), a good approximation may be derived by internal force $\overrightarrow{\mathbf{e}}(\overrightarrow{\mathbf{r}})=\delta \epsilon(\overrightarrow{\mathbf{r}}) / \delta \overrightarrow{\mathbf{r}}$ due to deformations on the object [8]:

$$
\overrightarrow{\mathbf{e}}(\overrightarrow{\mathbf{r}}) \cong \sum_{i, j=1}^{2}-\frac{\partial}{\partial a_{i}}\left(\alpha_{i j} \frac{\partial \overrightarrow{\mathbf{r}}}{\partial a_{j}}\right)+\frac{\partial^{2}}{\partial a_{i} \partial a_{j}}\left(\beta_{i j} \overrightarrow{\mathbf{n}}\right)
$$

where the terms $\partial\left(\beta_{i j} \vec{n}\right) / \partial r_{a_{1}}$ and $\partial\left(\beta_{i j} \vec{n}\right) / \partial r_{a_{2}}$ were disregarded and

$$
\begin{aligned}
\alpha_{i j}(\vec{a}, \vec{r}) & =\eta_{i j}(\vec{a})\left(G_{i j}-G_{i j}^{0}\right) \\
\beta_{i j}(\vec{a}, \vec{r}) & =\xi_{i j}(\vec{a})\left(B_{i j}-B_{i j}^{0}\right) .
\end{aligned}
$$

Further, Terzopoulos et al. used the second derivatives $\partial^{2}(\overrightarrow{\mathbf{r}}) / \partial a_{i} \partial a_{j}$ as an approximation to the normal vector $\vec{n}$ in Eq. (18) and obtained the following expression

$$
\overrightarrow{\mathbf{e}}(\overrightarrow{\mathbf{r}}) \cong \sum_{i, j}-\frac{\partial}{\partial a_{i}} \cdot\left(\alpha_{i j} \frac{\partial \overrightarrow{\mathbf{r}}}{\partial a_{j}}\right)+\frac{\partial^{2}}{\partial a_{i} \partial a_{j}}\left(\beta_{i j} \frac{\partial^{2} \overrightarrow{\mathbf{r}}}{\partial a_{i} \partial a_{j}}\right),
$$

Since quantities $G_{i j}$ are related to surface stretching and shearing, while the values for $B_{i j}$ are related to bending and twisting, our measures of deformation follow from these quantities and the surface's behavior of resistance to external forces will be as much effective as greater are the values assigned to the elasticity parameters.

Observing Eqs.(16) and (17) we will see that the geometric shape of a deformable surface can be controlled not
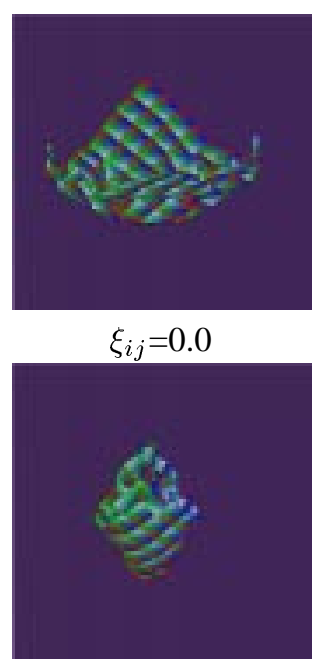

$\xi_{i j}=3.6 \times 10^{-7}$

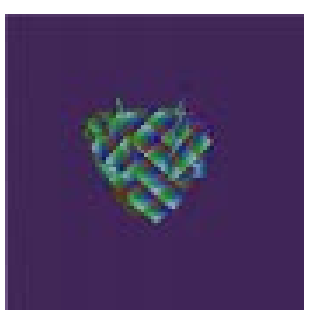

$\xi_{i j}=8.0 \times 10^{-8}$

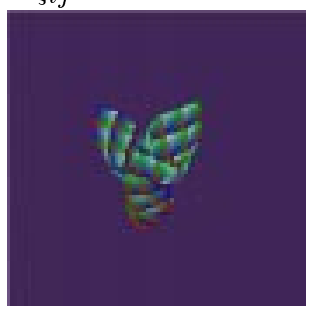

$\xi_{i j}=7.0 \times 10^{-7}$
Figure 5: Distinct behaviors towards curving resistance.

only by $\mu, \gamma$ and external forces $\vec{f}(\vec{r}, t)$, but also by the elasticity parameters. Due to the diversity of parameters involved, different combinations of those parameters could take us to the same visual effect.

That flexibility increases the model's versatility, but, on the other hand, makes it difficult to be controlled, since these parameters are not orthogonal and the influence of some parameter values can be masked by others. Ramos et al. proposed the distinction of two control levels [11]:

Rigid-body motion control: By assigning adequate values for $\mu, \gamma$ and $\vec{f}$, we may have an inaccurate, but intuitive control of the object's dynamics. All points in the surface assume equal values of $\mu$ and $\gamma$, considering that the surface is homogeneous in relation to the environment in which is immerse.

Pure deformation control: The variations in the local geometry of each point is caused by the material's resistance to variations in stretching and bending. That effect can be controlled by properly setting the elasticity parameters $\eta_{i j}$ and $\xi_{i j}$.

On the basis of the parameter choices suggested by Ramos et al., we carried out a set of simulations in the deformable model given by Eq. (21) and group them into four classes of deformations:

1. no resistance to metric variation and no resistance to curving $\left(\eta_{i j} \approx 0\right.$ and $\left.\xi_{i j} \approx 0\right)$ such as the effect of gravity force acting on a piece of melting cheese fixed at its boundary (Figure 1). Note that the deforming energy is completely absorbed by the points adjacent to the fixed ones; no deformation occurs in the interior of the object. 

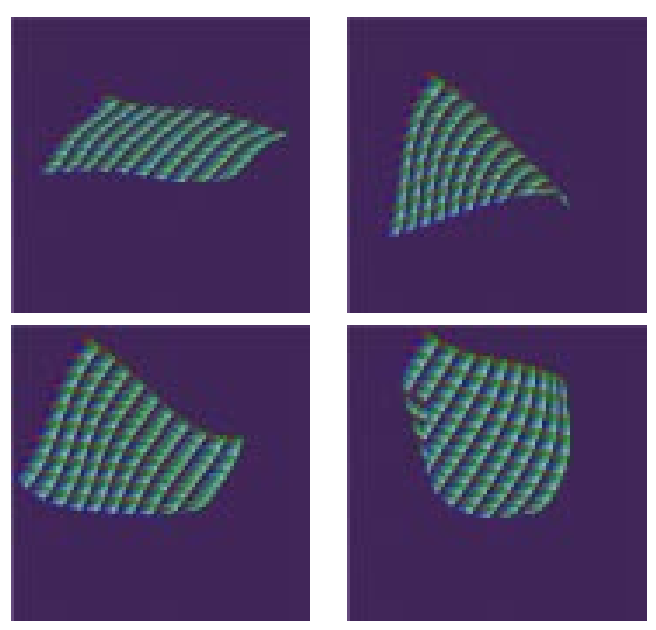

Figure 6: Effect of non-normalized normal vectors.

2. resistance to metric variation and no resistance to curving $\left(\eta_{i j} \neq 0\right.$ and $\left.\xi_{i j}=0\right)$ such as a piece of cloth under the action of the gravity force. Figure 2 illustrates a time sequence of a table cloth being spread on a table $\left(\eta_{i i}=0.001\right.$ and $\left.\eta_{12}=\eta_{21}=0.0006\right)$. In this case, variations on the area of the deforming object cannot be perceived visually.

3. resistance to metric variation and no resistance to curving $\left(\eta_{i j} \neq 0\right.$ and $\left.\xi_{i j}=0\right)$ such as a rubber sheet with fixed boundary under the action of the gravity force, as depicted in Figure 3. Note that as the value of $\eta_{i j}$ increases, the sheet tends to remain flat once it offers more resistance to stretching. The perception of "variation in bending" is due to the natural "geometric compatibility adjustment" since there is no resistance to curving and the border is fixed.

4. resistance to metric variation and resistance to curving $\left(\eta_{i j} \neq 0\right.$ and $\left.\xi_{i j} \neq 0\right)$ such as a piece of $\operatorname{cardboard}\left(\eta_{i j}=\right.$ 0.01 and $\xi_{i j}=1.0 \times 10^{-8}$ ) under a pressing force at its center, as shown in Figure 4.

From our experiments, we noticed that it is difficult to control the bending deformation with the elasticity parameters $\xi_{i j} \neq 0$. Most of visual results do not correspond to the expected physical behavior. Figure 5 gathers the simulation results of a piece of paper for the same set of simulation parameters, except the resistance to curving under a force applied at its center. Observe that for a fixed $\eta_{i j}$, when the value of $\xi_{i j}$ increases, the sheet of paper tends to fold, instead of maintaining flat. It seems that curving resistances spontaneously generate additional forces to produce twisting effect. Our conjecture is that these unrealistic effects are due to the lack in the control of the relationship between the metric and curvature tensors while the surface is deforming.
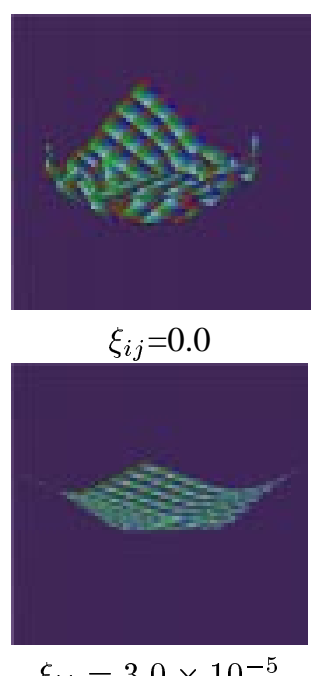

$\xi_{i j}=3.0 \times 10^{-5}$

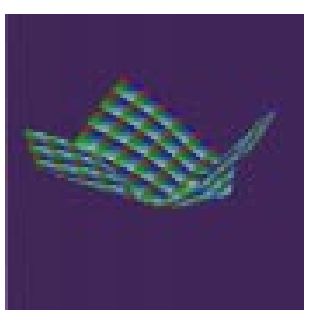

$\xi_{i j}=5.0 \times 10^{-6}$

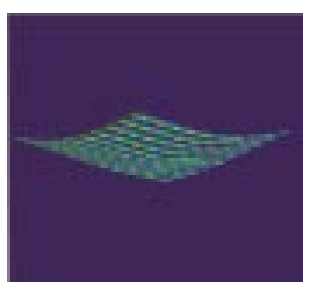

$\xi_{i j}=1.0 \times 10^{-4}$
Figure 7: Distinct behaviors towards curving resistance in a new approach.

\section{A new approximation for $\vec{n}$}

As shown by Eq.(21), Terzopoulos et al. [12] approximate the normal vector $\overrightarrow{\mathbf{n}}$ that appears in equation (18) by the second derivatives. This approximation may distort not only the magnitude but also the direction and sense of curving deformations, since the directions of the second derivatives do not necessary coincide with the direction of the normal vector $\overrightarrow{\mathbf{n}}$. Therefore, unexpected bending effects may be yielded. In this section, we present an alternative approximation for the normal vector $\overrightarrow{\mathbf{n}}$ in Eq.(19). In our approximation, the main concern is that the components of the metric and the curvature tensors must satisfy the compatibility relation expressed by Eq. (9) and that the result is easily linearizable.

Using Eq.(7), the following approximation is derived

$$
\begin{aligned}
\frac{\partial^{2}}{\partial a_{i} \partial a_{j}}\left(\beta_{i j} \overrightarrow{\mathbf{n}}\right) & =\frac{\partial}{\partial a_{i}}\left(\frac{\partial}{\partial a_{j}} \beta_{i j} \overrightarrow{\mathbf{n}}\right) \\
& =\frac{\partial^{2} \beta_{i j}}{\partial a_{i} \partial a_{j}} \overrightarrow{\mathbf{n}}+\sum_{k=1}^{2} \frac{\partial \beta_{i j}}{\partial a_{j}} d_{k i} \frac{\partial \overrightarrow{\mathbf{r}}}{\partial a_{k}} \\
& +\frac{\partial}{\partial a_{i}}\left(\beta_{i j} \sum_{k=1}^{2} d_{k j} \frac{\partial \overrightarrow{\mathbf{r}}}{\partial a_{k}}\right)
\end{aligned}
$$

We would like to write an expression for $\overrightarrow{\mathbf{n}}$ in terms of the derivatives of $\overrightarrow{\mathbf{r}}$, without resorting to cross product in order to also make possible the transformation of the system of partial differential equations (19) into a system of ordinary differential equations. From Eq.(9), we have at each non-singular point $\left(B_{i j} \neq 0\right)$

$$
\overrightarrow{\mathbf{n}}=\frac{1}{B_{i j}}\left[\frac{\partial^{2} \overrightarrow{\mathbf{r}}}{\partial a_{i} \partial a_{j}}-\Gamma_{i j}^{1} \frac{\partial \overrightarrow{\mathbf{r}}}{\partial a_{1}}-\Gamma_{i j}^{2} \frac{\partial \overrightarrow{\mathbf{r}}}{\partial a_{2}}\right] .
$$



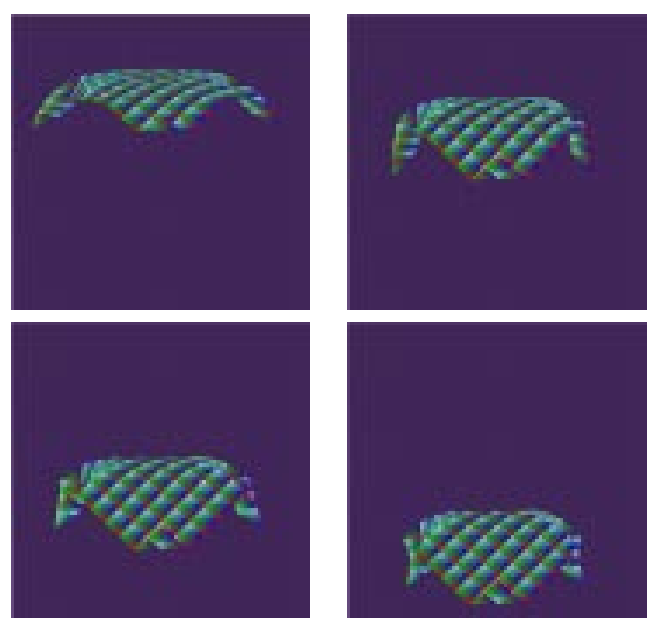

Figure 8: Effect of normalized normal vectors.

The Christoffel symbols $\Gamma_{i j}$ may be computed from the coefficients of the metric tensor and their derivatives with the help of Eq.(15), while the components of the curvature tensor $B_{i j}$ depend on the second derivatives and the normal vector to be calculated as shows Eq.(6).

To simplify, we used the fact that the surface is continuously deforming and the time and spatial discretizations are sufficiently fine that there is no abrupt modification in the normal vectors and in the Christoffel symbols and in $B_{i j}$ at any point of the surface between two subsequent states $k-1$ and $k$ of a time sequence of deformable models. At each state $k, B_{i j, k-1}$ is determined from the normal vector $\overrightarrow{\mathbf{n}}=\left(\frac{\partial \mathbf{r}_{\mathbf{k}-1}}{\partial a_{1}} \times \frac{\partial \mathbf{r}_{\mathbf{k}-\mathbf{1}}}{\partial a_{2}}\right) /\left\|\frac{\partial \mathbf{r}_{\mathbf{k}-\mathbf{1}}}{\partial a_{1}} \cdot \frac{\partial \mathbf{r}_{\mathbf{k}-\mathbf{1}}}{\partial a_{2}}\right\|$ as expressed by Eq. 6 and is applied together with $\Gamma_{i j, k-1}^{1}$ and $\Gamma_{i j, k-1}^{2}$ at the state $k-1$ to compute the normal vector $\overrightarrow{\mathbf{n}}_{k}$ for the state $k$

$$
\overrightarrow{\mathbf{n}}_{k}=\frac{1}{B_{i j, k-1}}\left[\frac{\partial^{2} \overrightarrow{\mathbf{r}}_{k}}{\partial a_{i} \partial a_{j}}-\Gamma_{i j, k-1}^{1} \frac{\partial \overrightarrow{\mathbf{r}}_{k}}{\partial a_{1}}-\Gamma_{i j, k-1}^{2} \frac{\partial \overrightarrow{\mathbf{r}}_{k}}{\partial a_{2}}\right] .
$$

In principle, only the direction of normal vectors is important for determining the bending deformation energy, our first attempt was ignoring the norm of $\overrightarrow{\mathbf{n}}$ by setting $\left|B_{i j}\right|=1.0$. However, some non-realistic visual effects were still observed. Figure 6 illustrates, after a large reaction force, how the deforming surface may suffer displacements in the tangential direction in such a way that it seems "rotating", even when only forces in the normal direction are applied. Tracing the simulation procedure, we observed that the norm of a non-normalized normal vector may be very large and may contribute unrealistically to the summation of the deforming forces.

If we normalize the normal vector, when the spatial discretization is coarse we still faced cases for which $B_{i j}$ is so small that $\overrightarrow{\mathbf{n}}_{k}$ may suffer from numerical imprecision.

As an intermediary solution, we opted for estimating
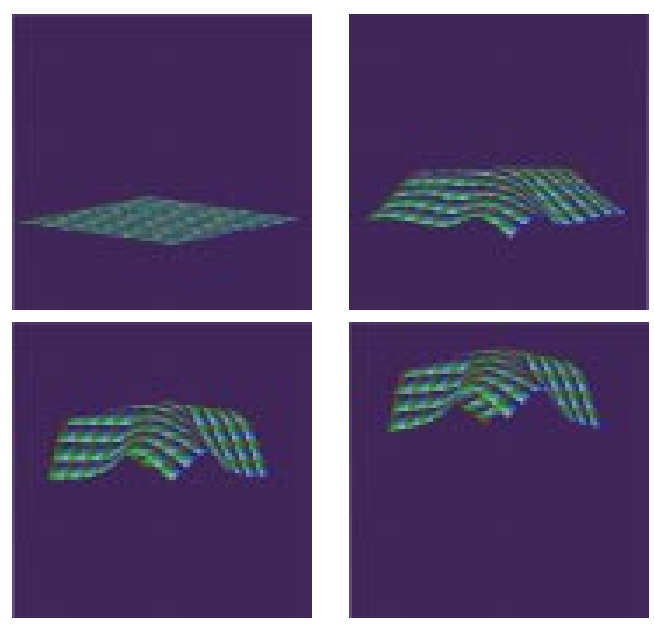

Figure 9: Lifting a cardboard.

$\overrightarrow{\mathbf{n}}_{k}$ with the following expressions, where $\delta$ is a function of the initial spatial discretization of the mesh:

- if $\left|B_{i j, k-1}\right|>\delta$, then

$$
\frac{1}{B_{i j, k-1}}\left[\frac{\partial^{2} \overrightarrow{\mathbf{r}}_{k}}{\partial a_{i} \partial a_{j}}-\Gamma_{i j, k-1}^{1} \frac{\partial \overrightarrow{\mathbf{r}}_{k}}{\partial a_{1}}-\Gamma_{i j, k-1}^{2} \frac{\partial \overrightarrow{\mathbf{r}}_{k}}{\partial a_{2}}\right]
$$

- if $0.0<B_{i j, k-1}<\delta$, then

$$
\left[\frac{\partial^{2} \overrightarrow{\mathbf{r}}_{k}}{\partial a_{i} \partial a_{j}}-\Gamma_{i j, k-1}^{1} \frac{\partial \overrightarrow{\mathbf{r}}_{k}}{\partial a_{1}}-\Gamma_{i j, k-1}^{2} \frac{\partial \overrightarrow{\mathbf{r}}_{k}}{\partial a_{2}}\right]
$$

- if $-\delta<B_{i j, k-1}<0.0$, then

$$
-\left[\frac{\partial^{2} \overrightarrow{\mathbf{r}}_{k}}{\partial a_{i} \partial a_{j}}-\Gamma_{i j, k-1}^{1} \frac{\partial \overrightarrow{\mathbf{r}}_{k}}{\partial a_{1}}-\Gamma_{i j, k-1}^{2} \frac{\partial \overrightarrow{\mathbf{r}}_{k}}{\partial a_{2}}\right] .
$$

Experimentally, we observed that $\delta=0.5$ delivers best results for the cases when the discretization is such a one that the mapping of each of its cells is a almost planar patch in $R^{3}$.

Standard finite difference approximation as suggested by Terzopoulos et al., is used to discretize Eq.(22) for getting

$D_{i j}^{-}\left(\beta_{i j}\right) \overrightarrow{\mathbf{n}}+\sum_{k=1}^{2} D_{j}^{-}\left(\beta_{i j}\right) d_{k i} D_{k}^{+}(\overrightarrow{\mathbf{r}})+D_{i}^{-}\left(\beta_{i j} \sum_{k=1}^{2} d_{k j} D_{k}^{+}(\overrightarrow{\mathbf{r}})\right)$,

where $\overrightarrow{\mathbf{n}}$ is computed from the discretization of the expressions (24-26). The discretization of the common part of three equations is

$$
\left[D_{i j}^{+}(\overrightarrow{\mathbf{r}})-\Gamma_{i j}^{1} D_{1}^{+} \overrightarrow{\mathbf{r}}-\Gamma_{i j}^{2} D_{2}^{+} \overrightarrow{\mathbf{r}}\right] .
$$

Some terms are not computable at the grid boundary points. Knowing that the normal vector must be perpendicular to the tangent plane, if the term $D_{i j}^{+}(\overrightarrow{\mathbf{r}})$ cannot be determined, we also set $\Gamma_{i j}^{1} D_{1}^{+} \overrightarrow{\mathbf{r}}=\Gamma_{i j}^{2} D_{2}^{+} \overrightarrow{\mathbf{r}}=0$ to ensure that $\overrightarrow{\mathbf{n}}$ does not lie on the tangent plane. 

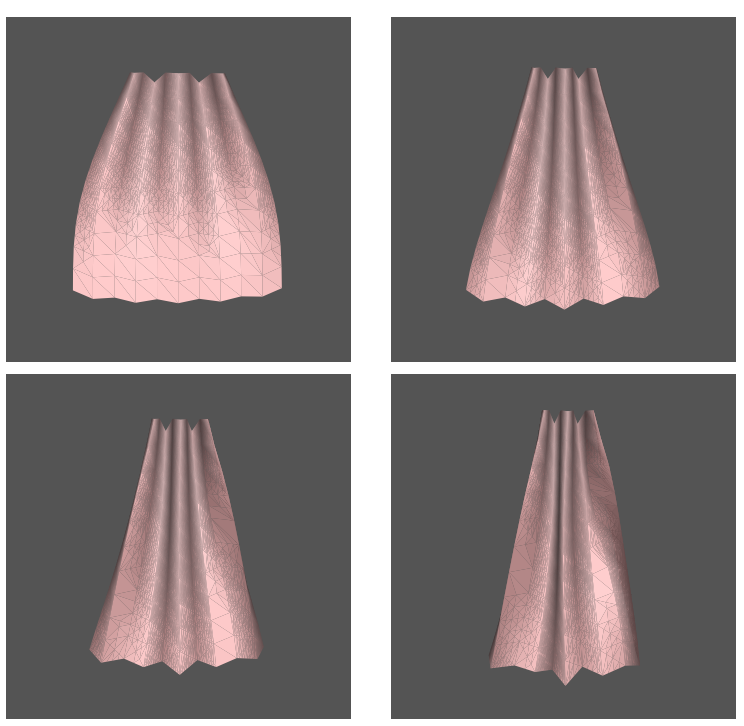

Figure 10: Opening a woolen curtain.

\section{New Simulation Results}

In this section we present some representative simulation results obtained with our proposed approximation for the normal vectors and the term $\frac{\partial^{2}}{\partial a_{i} \partial a_{j}}\left(\beta_{i j} \overrightarrow{\mathbf{n}}\right)$.

Figure 7 shows the new simulation results with the same set of simulation parameters used in Figure 5. Observe that when the resistance to curving increases, the sheet of paper tends to preserve its flatness instead of folding.

Figure 8 shows the results obtained with the same simulation parameters we used for the animation sequence presented in Figure 6. Note that the visual effect we obtained in Figure 8 is closer to our intuition than the one presented in Figure 6.

Figure 9 illustrates a simulation of lifting a sheet of cardboard which offers a big resistance both to stretching $\left(\eta_{i j}=1.0 \times 10^{-2}\right)$ and curving $\left(\xi_{i j}=2.0 \times 10^{-6}\right)$. The lifting force is applied at the middle point.

Figure 10 presents a simulation of opening of a woollen curtain $\left(\eta_{i i}=1.0 \times 10^{-3}, \eta_{12}=\eta_{21}=1.0 \times 10^{-6}\right.$ and $\xi_{i j}=2.0 \times 10^{-6}$ ) by applying the same force along its upper line.

Figure 11 shows a simulation of opening of a silk curtain that differs from the previous one only in the $\xi_{i j}$. In this simulation, $\xi_{i j}=0.0$

Figure 12 presents the results of a simulation similar to the one illustrated in Figure 2. We only alter the value of curving resistance $\left(\xi_{i i}=1.0 \times 10^{-5}\right.$ and $\xi_{12}=\xi_{21}=$ $1.0 \times 10^{-7}$ ). Observe that with resistance to curving, the behavior of the table cloth turns from the fabric material to the cardboard one.

Despite of the good visual simulation results from this section, in which we used distinct values for the compo-
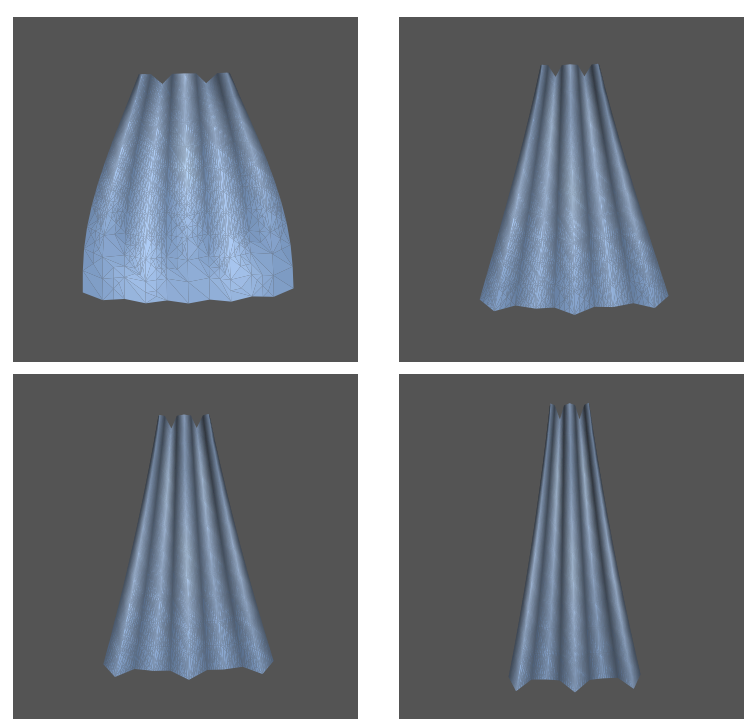

Figure 11: Opening a silk curtain.

nents of $\eta$ and $\xi$ to obtain the shearing and twisting effects, the relation between the values of $\xi_{i j}, i \neq j$ and twisting effects does not correspond to our expectation yet in most experiments we performed.

\section{Concluding Remarks}

The expected visual effect of the curving resistance forces is to act against the external forces in the normal vector direction. More reliable are the normal vectors, more realistic are the visual effects. We present another expression for computing the term $\frac{\partial^{2}}{\partial a_{i} \partial a_{j}}\left(\beta_{i j} \overrightarrow{\mathbf{n}}\right)$ in Eq.(19) and an approximation for the normal vectors on the basis of Gauss formulae which take the compatibility of the metric and the curvature tensors into consideration. Much more intuitive results have been achieved with small increment in the computational complexity. Further investigation of the relationship between the parameter $\delta$ and a spatial discretization is required to provide an adaptative meshing procedure.

In our tests, we perceived that the control of twisting is still awkward. When $\xi_{i j} \neq 0, i \neq j$, excessive displacements in unexpected directions can occur. We believe that the problem may rely on the deformation model proposed by Terzopoulous and we plan to investigate alternative models that also relate the physical variables with the geometric measurements.

\section{Acknowledgments}

We acknowledge the financial support to the second author provided by CAPES/PICDT during his staying at Unicamp while this work is performed for his Doctorate. 

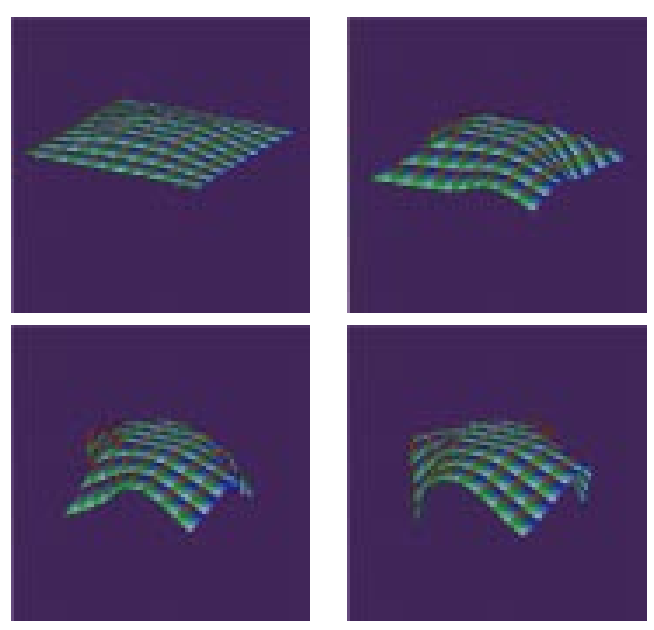

Figure 12: Resistance to metric variation and resistance to curving.

\section{References}

[1] Breen, D.E., House, D.H., and Getto, P.H..A PhysicalBased Particle Model of Woven Cloth. Visual Computer, Vol.8, 1992, pp. 264-277.

[2] Carignan, M., Yang Y., Thalmann, N. M. and Thalmann D.. Dressing Animated Synthetic Actors with Complex Deformable Clothes. Computer Graphics, 1992, Vol.26, No.2, pp.99-104.

[3] Carmo, M.P.. Differential Geometry of Curves and Surfaces. Prentice Hall Englewood Cliffs, N.J., 1976.

[4] Celniker, G. and Grossard, D.. Deformable Curve and Surface Finite-elements for Free-form Shape Design. Computer Graphics, 25(4):257-266, July 1991.

[5] Eischen, J. W., Deng, S., and Clapp, T.G.. FiniteElement Modeling and Control of Flexible Fabric Parts. IEEE Computer Graphics and Applications, 1996, pp. 71-80.

[6] Feyman, C.R.. Modelling the Appearance of Cloth. MSc Thesis, Department of Electrical Engineering and Computer Science, MIT, Cambridge, MA, 1986.

[7] Green, A.E. and Zerna, W. .Theoretical Elasticity. Second Edition, 1968. Oxford University Press.

[8] Horta, A.A., Wu, S.T.. Deformação de Superfícies Não Rígidas Baseada em Princípios Físicos. Anais do VIII SIBGRAPI, 1995, pp.175-182.

[9] Kreyszig, Erwin. Differential Geometry. Dover Publications, Inc., New York, 1991.
[10] Provot, X.. Deformations Constraints in a Massspring Model to Describe Rigid Cloth Behavior. Proc. of Computer Graphics Interface, 1995, pp. 147-154.

[11] Ramos J., P.F.S.C., Wu, S.T, Costa,S.I.R.. Analyzing a Deformable Model Using Differential Geometry. Anais do X SIBGRAPI, 1997, pp.57-64.

[12] Terzopoulos, D., Platt J., Barr A. and Fleischer K.. Elastically Deformable Models. SIGGRAPH'87, Computer Graphics, 1987, Vol.21, No.4, pp.205-214.

[13] Weil, J.. The synthesis of cloth objects. Computer Graphics, 1986, Vol.20, No.4, pp.49-54.

[14] Wu, Y., Thalmann, D., Thalmann, N. M. Deformable Surface Using Physically-Based Particle Systems, Proc. Computer Graphics International, Leeds, Academic Press, 1995, pp. 205-216. 